Aus der chirurgischen Klinik in Innsbruck.

(Vorstand: Prof. v. Haberer.)

\title{
Zur Frage nach dem Operationsverfahren beim perforierten Magen-Duodenalgeschwür.
}

\author{
Von Dr. Ernst Paul, Assistent der Klinik.
}

Wenn in der operativen Behandlung des Ulcus ventriculi und duodeni trotz eifrigen Arbeitens auf klinischem und pathologischanatomischem Gebiete und vielfacher Verbesserungen in der operativen Technik noch keine Entscheidung darin erfolgt ist, ob den direkten vor den indirekten Methoden der Vorzug zu geben sei, so lagen für das Ulcus perforatum die Dinge so, daß die Resektionsmethode bis vor kurzem überhaupt nicht als Verfahren ernstlich in Betracht gezogen wurde. Denn bei voller Übereinstimmung über die Notwendigkeit aktiven Vorgehens in der Therapie dieser Peritonitisform war der bisherige Standpunkt durch die allgemein geltende Regel präzisiert, daß die Laparotomie durch keinerlei Maßnahmen verlängert und kompliziert werden darf, die nicht direkt bezwecken, den unmittelbaren Gefahren der Peritonitis entgegenzuwirken, den Kranken vor drohender Lebensgefahr zu retten. Nur die Naht in magenquerer Richtung kam deshalb bisher als zweckmäßiger und kürzester Eingriff in Betracht. Für jene Fälle, bei denen die Ausführung dersèlben auf unüberwindliche Schwierigkeiten stieß (Durchschneiden der Nähte, ausgedehnte Infiltration), waren Methoden empfohlen, die gleichsam als Aushilfen entweder fremdes Material aus der Umgebung (Braun, Bennet u. a.) zum Schlusse der Öffnung benutzten, oder die Isolierung derselben von der freien Bauchhöhle zum Ziele hatten; ob das jetzt unmittelbar (Burk) durchgeführt wird oder mittelbar durch Benutzung der Perforationsstelle zur Anlegung einer Gastrostomie, ist ohne wesentliche Bedeutung. Zu erwähnen wäre noch, daß sich zur Sicherung einfach übernähter Perforations- 
öfnungen die Hinzufügung der Jejunostomie (v. Eiselsberg) gut bewährt hat.

Doch das Auftreten von Verengerungen des Duodenallumens bzw. Magenausganges infolge Übernähung und Einstülpung perforierter Pylorus- bzw. Duodenalulcera machte die Frage, ob eine Gastroenterostomie prinzipiell anzuschließen (Schloffer, Hochenegg, Körte, Demmer u. a.) oder entbehrlich sei (Noetzel, Petrén, Moynihan) zum Gegenstande eingehender Erörterung. Während die einen in der gleichzeitigen Anlegung der G. E. eine Entlastung der Naht, damit die Möglichkeit früher Nahrungszufuhr als Vorteil erblickten oder durch die Änderung des Magenchemismus einen günstigen Einfluß auf die Heilung des Geschwüres selbst erwarteten, wollten die anderen sie nur dann angewendet wissen, wenn ihre Anlegung dringend geboten und die Aussichten des Patienten die Perforationsperitonitis zu überleben, dadurch vermehrt seien. Dem einen Teile der Chirurgen erschien es gewagt, darüber hinauszugehen, was die augenblickliche Lebensgefahr zu tun fordert, der andere verlangte die Durchführung von Maßnahmen, die durch Schaffung günstigerer Heilungsbedingungen das Grundleiden beeinflussen sollten.

Die Erfahrung brachte die Kenntnis, daß die Übernähung nicht immer als eine absolut sichere anzusehen sei, daß sie exakt durchgeführt zu Stenosen führe, die Nachoperationen notwendig mache und ferner, daß die aus mechanischen und chemischen Gründen angelegte G.E. keineswegs immer zur Ausheilung des Ulcus führen müsse, deshalb auch nicht vor seinen lebensbedrohlichen Komplikationen wie Blutung und neuerliche Perforation schützen könne. Erst die Kenntnis von der Multiplizität der Ulcera hat das Auftreten der Komplikationen bei übernähten und gastroenterostomierten Ulcusperforationen in ein anderes Licht gerückt.

Eine weitere gefährliche Komplikation stellte das Ulcus pepticum jejuni dar, dessen Auftreten technisch nicht zu vermeiden ist.

Nach den hier nur kurz angeführten Möglichkeiten k a n $\mathrm{n}$ ein nach Ulcusperforation Gastroenterostomierter wegen Folgezuständen von seiten des unbeeinflußt gebliebenen Geschwüres weiterhin in Gefahr schweben. Wann dies der Fall ist, und warum bei dem einen Ulcus die G.E. günstig wirkt, beim anderen nicht, ist uns bisher noch dunkel. Zur Bestätigung der obigen Behauptungen 
sei die Arbeit Brütts angezogen, der an dem Material der Kümmellschen Klinik, die anläßlich der Nachuntersuchungen gefundenen Neuerkrankungen bei C̈bernähung und G. E. mit 12 Proz. bemessen konnte, und in 36 Proz. der ad exitum gekommenen Geschwürsperforationen multiple Uilcera fand.

Trotzdem sich die Resektionsmethode schon infolge ihrer größeren Radikalität als viel sicheres Verfahren in der Behandlung des Lilcus ventriculi et duodeni erwies und dabei Zustände, wie sie im Gefolge der G. E. beobachtet werden, doch vicl seltener wahrzunehmen sind, konnte sie sich als Methode der Wahl noch nicht allgemein durchsetzen ${ }^{1}$ ); neben dem Unvermögen kausaler Therapie wird ihr die Schwierigkeit der Ausführung und die dadurch höhere Mortalität (10 Proz.) als dem Leiden nicht entsprechend zum Vorwurf gemacht. Die Verwendung der Resektion beim perforierten Ulcus wird hauptsächlich noch aus dem Grunde abgelehnt, weil der Allgemeinzustand des Patienten doch gewöhnlich ein sehr elender ist, andererseits die dabei an das Peritoneum gestellten Anforderungen als zu hoch erschienen. Deshalb sind in der Literatur der früheren Jahre auch nur wenige Fälle von Resek. tion bei perforiertem Ulcus $\mathrm{zu}$ finden ( $\mathrm{Hahn} \mathrm{I902,} \mathrm{Tobin,}$ Keetly Igor, Maunsell, Petrén I907, Propping 1913) ${ }^{2}$, dic eben nur vereinzelte $V$ ersuche geblieben sind. Es mußte deshalb dic Anregung, durch die $\mathrm{Haberer}$ auf (irund zweier durch Resektion bzw. Pylorusausschaltung geheilter Perforationen die Resektionsmethode beim durchgebrochenen Geschwür in den Bereich ernstlicher Erwägung zog, zur $\mathrm{Nachprüfung}$ herausfordern. Unabhängig von $\mathrm{ihm}$ hat $\mathrm{M}$ assari die Resektion in günstig gelegenen Fällen mit gutem Resultate ausgeführt; in rascher Folge sind gute Operationsresultate nach Resektion des die Perforation tragenden Magenteiles zur allgemeinen Kenntnis gelangt. Nebenstehende Tabelle, welche die bisher veröffentlichten Resektionsfälle berücksichtigt, ergibt deutlich, wie jung dieses Verfahren beim Ulcus perforatum ist.

I) Vergleichsstatistiken über Dauerresultate nach G. E. und Resektion beim Ulcus ventriculi im allgemeinen geben die Arbeiten von $\mathrm{E}$. Hempel. Inaug. L)iss. Locij.zig 1021, und E. Jüngermann, Deutsche Zuitschr. f. Chir. $1920, \mathrm{Bd} . \mathrm{I} 58$.

2) Zitiert nach Fr. Brunner, Petrén u. Demmer. 


\begin{tabular}{|c|c|c|c|c|c|c|}
\hline Autoren & $\begin{array}{c}\text { Ulcus } \\
\text { duodeni }\end{array}$ & $\begin{array}{l}\text { Ulcus } \\
\text { pyloric. }\end{array}$ & $\begin{array}{c}\text { Ulcus } \\
\text { ventriculi }\end{array}$ & Spïlung & Trocken & $\begin{array}{l}\text { Todes- } \\
\text { fälle }\end{array}$ \\
\hline Haberer & - & - & I & I & - & $\ldots$ \\
\hline Prader. & 5 & 一 & - & - & 5 & - \\
\hline Eunike. & - & 2 & 1 & - & 3 & - \\
\hline Hromada. & 6 & 1 & 3 & 13 & - & 4 \\
\hline Massari. & - & 2 & - & - & 2 & - \\
\hline Schwarzmann & 3 & 4 & 1 & I & 7 & \\
\hline Eberle. . & - & - & I & I & - & -- \\
\hline Marconi. & - & - & $i$ & - & i & - \\
\hline Dewes. & - & - & I & I & - & - \\
\hline
\end{tabular}

Nachfolgenden Ausführungen liegt das Material der an der chirurgischen Klinik zu Innsbruck und Privatklinik Prof. v. Ha berers operierten Magen-Duodenalperforationen seit dem Jahre 1919 zugrunde. Die Wahl dieses Zeitabschnittes dürfte den Anschein der Willkür erwecken, ist aber dadurch begründet, daß die Methode erst im Jahre I9I9 ausgeführt wurde und die Arbeit den $Z$ weck verfolgt, an einer fortlaufenden Reihe einheitlich behandelter Fälle den Wert der beiden Operationsverfahren an dem Verhältnis ihrer Operations- und Dauerresultate zu messen. Dazu brachte uns die Kriegszeit merkwürdigerweise eine Verminderung an diesem Material, die nur so erklären ist, daß bei der großen Zahl von Spitälern (Innsbruck lag im Etappenbereich) die Perforationen an Ort und Stelle versorgt und nicht erst transportiert wurden; weiter sind Nachuntersuchungen aus diesem Zeitraum dadurch erschwert, daß die meisten Patienten nicht mehr eruierbar oder gefallen sind.

Wir sind uns von vornherein darüber klar, daß es bei dem Mißtrauen, das derartigen Statistiken mit Recht entgegengebracht wird, schwer ist, Andersdenkende zu überzeugen und halten es deshalb für die Beurteilung der Heilungserfolge notwendig, den Standpunkt der Innsbrucker Klinik in der Frage der Peritonitisbehandlung, hauptsächlich ob Spülung oder Austupfen, Drainage oder primärer Schluß der Bauchwunde anzuwenden sind, zu präzisieren.

Haberer und $\mathrm{Chiari}$ haben dieses Thema schon eingehend behandelt, so daß Schlagworte hier genügen dürften. Dort, wo es sich um eine progrediente, allgemeine Form der eitrigen Peritonitis handelt, wird gespült. Die allgemein bekannte Schwierig. 
keit aus der freien Peritonealhöhle Eiter oder Verschmutzung rest los zu entfernen und die Unmöglichkeit einer vollständigen, genügend lange wirksamen Drainage der freien Bauchhöhle lieb uns zu jenem Verfahren greifen, das bei geringster Serosaschädigung die bestmöglichsten Resultate erzielen läßt. Das Peritoneum muß mit dem Reste infekiösen Materiales fertig werden, um so mehr, als die Infektiosität, wie schon von $\mathrm{C}$. Brunner nachgewiesen und in der letzten Zeit auch von $\mathrm{Pracler}$ wieder an Io Fällen festgestellt wurde, beim licus ventriculi und duodeni perf. durchschnittlich keine sroße ist im Vergleich zu den Peritonitiden aus anderer I'rsache. Am günstigsten lagen jene Fälle, die innerhalb der Zeit, in welcher alkalische Imstimmung des bakterienführenden Magensaftes noch nicht vollendet war, zur Operation kamen. Die Spülung wurde in der von $\mathrm{Haberer}$ (Wienerklin. Wochenschr. 1920! angegebenen Weise sofort nach Eröfnungr der Peritonealhöhle eingeleitet und währte während der ganzen \%eit der operativen Maßnahmen. Durch mehrmaliges Wechseln des $\mathrm{Zu}$. laufrohres wird der Flüssigkeitsstrom in verschiedenen Richtungen rurchgetrieben, so daß nach Beendigung der Operation das Spülwasser klar abfließen mulBte. Besondere Aufmerksamkeit wurde der speriellen Reinigung des (Jberbauches, der Milz und Leber. nische geschenkt. Bei in die Laparotomiewunde flach eingeführter Hand ward diese (iegend direkt mit Kochsalzlösung ausgewaschen und alle noch gröberen Teile mittels Kochsalzkompressen entfernt. Dadurch ist nicht nur jeder Zeitverlust vermieden worden, sondern auch die mit Rücksicht auf den Allgemeinzustand nicht ratsam erscheinende iventration dem I'at. erspart geblieben. Waren etwaige Adhäsionen gelöst, fand sich nirgends eine serosaentblößte Stelle (auf das ist besonderes Augenmerk zu richten!) und ist die Perforationsstelle entfernt oder geschlossen, dann darf man auch nach unseren Erfahrungen ruhig ohne I)rainage primär die Bauch. höhle schließen. Der primäre Schluß der Bauchwunde bei Peritonitis ist ja keineswegs etwas Neues! Nach Krohers historischem Rückblick wurde er schon viele Jahre vorher, aber nur fall- oder probeweise angewandt; später haben sich dann Körte, Schmid. Rotter, Nordmann u. a. dafür ausgesprochen und in letzterer Zeit hat die Methode immer mehr Anhänger gewonnen 
Ist dagegen die Peritonitis eine begrenzte, so beschränken wir uns auf das einfache sorgfältige Austupfen des Eiters und vermeiden die Spülung wegen Gefahr, das Material über noch nicht infizierte Teile der Bauchhöhle zu verschleppen; aber auch dann wird primär geschlossen, wenn nirgends nekrotisches Gewebe vorhanden und jede Blutung gestillt ist. Zeigt sich irgendwo ein serosaentblößter oder diffus blutender Raum, der durch Peritonisierung nicht aus der Welt geschaffen werden kann, oder handelt es sich um einen ausgesprochenen abgesackten Eiterherd, also einen AbszeB, dann wird drainiert! In allen Fällen ist der günstige Einfluß des primären Schlusses auf Atmung und Peristaltik unverkennbar. Einen Prolaps aus der Laparotomiewunde hatten wir nie zu verzeichnen. Nicht unwichtig erscheint es uns darauf hinzuweisen, daß man nicht immer berechtigt ist, selbst wenn sichtbare Adhäsionen älterer Art vorliegen, von einem abgegrenzten Prozeß zu sprechen. In einem Falle waren ausgebildete Adhäsionen als AbschluB gegen die unteren Darmpartien zu sehen und reichlicher Inhalt im Subphrenium rechts ließ im guten Glauben an die Begrenzung des Prozesses von einer Spülung Abstand nehmen. Die Obduktion ergab aber, daß Eiter hinter dem Colon ascendens unterhalb der Adhäsionen bereits bis in den Douglas gelangt war.

Der allgemein bekannten Tatsache, daß nach primärem Wundschluß der Bauchhöhle Infektionen des Subkutangewebes (auch Anaërobier) keine Seltenheit sind, wurde durch Verzicht auf die Hautnaht Rechnung getragen. Ohne wesentliche Temperatursteigerung und ohne klinische Erscheinungen verlaufend können derartige subfaszielle Eiterungen den Träger durch Einbrechen in die Peritonealhöhle in schwerste Lebensgefahr bringen. Tritt dieser besonders ungünstige Ausgang auch nur selten ein, so kommt es doch zu Fasciennekrose, langwierigen Eiterungen und unfehlbar zum Narbenbruch, mit dem der Grund zu so manchen lästigen Sensationen gelegt ist.

Keinen Fall haben wir an Komplikationen von seiten des Respirationstraktes verloren und abwohl alle in Äther-Tropfnarkose operiert wurden, blieben selbst Bronchitiden eine Seltenheit. Im postoperativen Verlaufe, der fast immer ein afebriler war, konnten wir die Beobachtung machen, daß das weiche Abdomen bis zum 3. Tage noch immer leicht empfindlich blieb; das ist vielleicht 
dadurch zu erklären, daß die Abwehrreaktion des Peritoneums, wenn auch schon dem Infekt überlegen, gegen den zurückgebliebenen Rest fortwährt. Die Darmtätigkeit setzte am 3. oder 4. Tage von selbst ein und blieb wciterhin ohne Nachhilfen geregelt.

Seit der Zeit vom Jahre I 919 wurden der Innsbrucker Klinik aus dem Stadt- und weiteren Umgebungsgebiete 27 Perforationen nach Magen-Duodenalgeschwüren überwiesen. Die auffallende Steigerung der Perforationsfälle im letzten Jahre auf das Doppelte ist einfach auf den Zufall zurückzuführen, daß der akute Durchbruch .Leute aus dem Stadtbereiche ereilte. Der Vorteil rascher Hilfeleistung geht der bäuerlichen Bevölkerung aus der Umgebung infolge der großen Entfernung vom Arzte und der Abhängigkeit von Weg- und Witterungsverhältnissen oft ab. Dadurch mag so màncher Fall von Magen-Duodenalperforation für die zahlenmäßige Behandlung verloren gehen und eine unbekannte Größe erstehen lassen, die das wirkliche Verhältnis zwischen Perforation und Vicera ïberhaupt nicht errechnen läßt.

Abgesehen von der zufäligen Zunahme im letzten Jahre ist auch ein absolutes Ansteigen der Zahl der Perforationsfälle gegenüber den Friedensjahren (auf das Doppelte) zu konstatieren, wie es auch von anderer Seite (Rosental, Baurmann) hervorgehoben wird. Vermutlich dürte diese Beobachtung Hand in Hand gehen, mit der Feststellung, daß nach dem Kriege die. Zahl der Ulcera eine bedeutende Vermehrung (auf das 4 fache) erfahren, die aber weniger als eine absolute Vermehrung (Best ${ }^{1}$ ) vielmehr als Exazerbation latent gebliebener Geschwüre (Albu, ( ra mer2) aufzufassen sei.

Ess findet sich ferner in dem Verhältnis zwischen den Perforationen des Magens und Duodenum eine merk würdige Verschiebung in dem Sinne, daß die Zahl der Duodenalperforationen sich immer mehr den früher fast um das Doppelte überwiegenden Magenperforationen nähert. Demmer, Petraschewskaja berech. nen das Verhältnis Ulcus ventriculi zum Ulc. duodeni wie $2: \mathrm{I}$, $\mathrm{Br}$ ütts 7ahlen entsprechen der Gleichung $\mathrm{I}: \mathrm{I}$ und die letzten Zusammenstellungen melden mehr Duodenal-als Magenulcera als perforiert; so Schülein I5:6, Roedelius I8: I5, Prader

1) 2) Zit. nach Baurmann. Deutsche Zeitschr. f. Chir., 154, S. 4r. 
$7: 4$, H r o m a da $6: 4$, B r ü tt 9:3. Unter unseren 27 Fällen finden sich 20 Duodenalulcera, deren Zurechnung zu diesen durch die Lage außerhalb der Pylorusvene (M a yo) und vor allem kaudal des Pylorusringes erfolgte. Dieses Überwiegen der Duodenalulcera als bloßen Zufall aufzufassen geht schon aus dem Grunde nicht an, weil die Zusammenstellung das Material dreier Jahre umfaßt. Ebensowenig scheint es mit der genaueren Lokalisationsbestimmung zusammenzuhängen; dürfte vielmehr damit in Einklang zu bringen sein, daß auch das übrige Ulcusmaterial der Klinik ein Ansteigen der Duodenalulcera aufweist.

Von den sieben perforierten Ulcera ventric. betrafen 3 Frauen (im 4. u. 5. Jahrzehnt). Das Alter der übrigen männlichen $\mathrm{Pa}$ tienten bewegte sich zwischen I9 und 67 Jahren. Nur nebenbei sei erwähnt, daß von den Duodenalperforationen bei Männern nicht weniger als I I das dreißigste Lebensjahr noch nicht erreicht hatten. Gleichlautend mit den Angaben von Schoenmaker, Demmer, Möller, Petrén war bei den Magenulcera ein Überwiegen des Sitzes im pylorischen Anteil zu konstatieren.

Dic Größe der Perforationsöffnung schwankte zwischen der eines Stecknadelkopfes und eines Hellerstückes, was für die Schwere der sich ausbildenden Peritonitis von Einfluß ist. Genügte auch der Austritt von minimalen Mengen Mageninhalt durch die feinste Öffnung zur Entstehung derselben, so ist der Durchtritt fester Speisebröckel durch den höheren Gehalt an Bakterien gefährlicher.

Die Zahl der wie gestanzt aussehenden Perforationsöffnungen mit normaler Umgebung ist klein; viel öfter wird eine ausgedehnte Infiltration der Ränder erwähnt. Bei der Bevorzugung der Vorderwand der kleinen Kurvatur des Magens und der Vorderseite des Duodenums kommt es in manchen Fällen zur Ausbildung einer förmlichen Narbenplatte, die nicht nur die Perforationsöffnung starr offen erhält, sondern auch der Versorgung derselben durch den Mangel normalen Gewebes bedeutende Schwierigkeiten in den Weg stellt. Das Duodenum ist durch derartige Narben, in die auch das Ligamentum hepatoduodenale einbezogen sein kann, stark geschrumpft, verkürzt und manchmal fest an die Hinterwand fixiert. So bleibt denn nach den vergeblichen Versuchen nichts anderes zu tun übrig, als fremdes Material aus der Umgebung dar- 
aufzunähen, sofern man sich nicht zur Resektion entschließen will oder kann. Manchmal läßt sich schon nach einigen präparatorischen Unterbindungen im Ligamentum hepatoduodenale ein oft überraschend langes Stück Duodenum entwickeln, das also mehr durch die Verwachsungen mit der Umgebung verkürzt erschien, als durch die schrumpfende Wirkung des Ulcus.

Der Befund eines $\mathrm{neben}$ dem perforierten Duodenalulcus bestehenden zweiten Geschwüres veranlaßt uns auf dieses Kapitel näher einzugehen. Wie schon oben bemerkt, ist das Vorkommen multipler Ulcera nicht selten, ihr Nachweis intra operationem aber manchmal sehr erschwert. Während uns früher die Kenntnis von der Multiplizität der pathologische Anatom brachte, ist in der Ära größerer Magenresektionen dieser Befund öfter erhoben worden. Es handelte sich dabei nicht immer nur um jene kleinen oberflächlichen Erosionen, sonđern um scharf begrenzte in die Submucosa vorgedrungene Ulcera, die gewöhnlich, aber durchaus nicht immer an der Aschoff-Waldeyerschen Magenstraße gelegen, sich gerne der Palpation entzogen, sei es, daß die Infiltration der Magenwand durch das größere Ulcus ein Tasten unmöglich machte oder daß mächtige Hypertrophie der Magenwand nichts Feineres unterscheiden ließ. Beim Ulcus duodeni liegen die Verhältnisse noch schwieriger. Bei Sitz eines solchen an der Vorderwand den Nachweis eines zweiten an der Hinterwand zu erbringen, ist trotz Mobilisation des Duodenums nach Kocher, wie sie $\mathrm{Ha}$ berer vorzunehmen pflegt, nicht immer möglich. Veränderungen der Serosa in Gestalt der weißlich schimmernden Sehnenflecke oder entzündlicher Rotfärbung können fehlen, Ligaturstümpfe können zu Täuschungen Veranlassung geben und um sicher zti gehen bleibt manchmal nichts anderes übrig, als die Duodenalklemme abzunehmen und die Schleimhaut zu revidieren. Aus diesen Beobachtungen geht hervor, da $B$ man bei der Beurteilung des Auftretens von Rezidiven nach Duodenalulcera sehr vorsichtig sein muß.

Bezugnehmend auf die statistischen Untersuchungen F. B r u n ners zur Ätiologie der Perforation mag Erwähnung finden, daß die meisten unserer Fälle (5) in dem Wintermonate Februar stattfanden, die nächst größere Zahl (4 Fälle) auf den Monat August traf; dieser Befund ist abweichend von dem $\mathrm{Br}$ u n. 
ners, der die meisten Perforationen auf die Monate Nov./Dez. oder Mai/Juni verteilt fand. Dieser Verschiebung dürfte bei der Kleinheit des Materiales keine weitere Bedeutung beizumessen sein.

An Hand der Fälle auf Symptomatologie, Diagnose und Differentialdiagnose des Ulcus perforatum näher einzugehen erscheint überflüssig, da weder Neues noch von dem bisher bekannten $\mathrm{Ab}$. weichendes beobachtet wurde. Das sicher nicht alltägliche Vorkommnis einer sozusagen unter den Augen des Chirurgen erfolgenden Perforation, die durch Hustenstöße zustande kam, mag kurz erwähnt sein. Äußere Momente sind ja nur ganz ausnahmsweise Ursache des Eintrittes der Perforation und in der Literatur zur Genüge bekannt (Petrén). Es soll hervorgehoben werden, daß gleich nach dem mit typischer Plötzlichkeit erfolgten Eintritte der Perforation die brettharte Bauchdeckenspannung und allgemeine Druckschmerzhaftigkeit des Abdomens

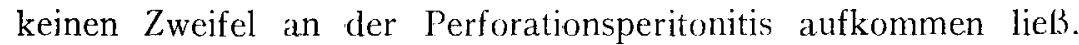
Die Diagnose kann also glejch gestellt werden und dadurch das weitere Handeln bestimmen. Damit soll keineswegs gesagt wer. den, daß über den Sitz der Perforation nicht auch einmal Unklarheit herrschen könne, wie das beim Fehlen einer ,Magenanamnese" und Bestehen eines Druckpunktes in der rechten Fossa iliaca der Fall sein kann. Derartige zweifelhafte Fälle sind unter unserem Material nur in der Dreizahl vorhanden. In allen anderen hat uns die ,kahnförmige Einziehung des Ober. ba uches" den sicheren Sitz der Perforation verraten. Es dürfte wenige Fälle geben, bei denen dieses Symptom nicht $z u$ beobachten ist; doch muß immer danach gefahndet werden, da es auch dann verwertbar erscheint, wenn es, wie manchmal, nur andeutungsweise vorhanden ist: Meist trat es so intensiv in Erscheinung, daß die Diagnose sofort gestellt werden konnte.

In bezug auf operative Maßnahmen verteilen sich die 27 Fälle auf I3 Resektionen, I I Gastroenterostomien nach Versorgung der Perforationsöffnung, zu denen wohl der eine Fall von Pylorusausschaltung gerechnet werden darf, und 3 einfache Übernähungen des Ulcus. Reseziert wurden I I Duodenal- und 2 Magenulcera, während mit Gastroenterostomie 9 Duodenal- und 2 Ulcera ventriculi behandelt wurden; die 3 einfachen Übernähungen kamen bei 


\begin{tabular}{|c|c|c|c|c|c|c|c|c|}
\hline 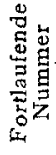 & $\mathrm{Name}$ & $\frac{\vec{y}}{\not{Z}}$ & 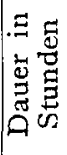 & $\begin{array}{c}\text { Sitz des } \\
\text { Ulcus }\end{array}$ & $\begin{array}{l}\text { Opera- } \\
\text { tions- } \\
\text { methode }\end{array}$ & $\begin{array}{l}\text { Behand- } \\
\text { lung der } \\
\text { Peritonitis }\end{array}$ & $\begin{array}{l}\text { Aus- } \\
\text { gang }\end{array}$ & $\begin{array}{c}\text { Ergebnisse } \\
\text { der } \\
\text { Nachuntersuchung }\end{array}$ \\
\hline I & Ma. Em. & 40 & $61 / 2$ & ventric. & QuereRes. & Spülung & Heilung & Beschwerdefrei \\
\hline 2 & Un. & 30 & 4 & pylor. & Res. B I. & $"$ & ", & $\begin{array}{l}\text { Nach einem Jahre } \\
\text { an intercurr. } \\
\text { Krankheit } t \text {. }\end{array}$ \\
\hline 3 & Jä. Pe. & 26 & 3 & duod: & Res. B I. & Trocken & , & $\begin{array}{l}\text { Schmerzfrei, iBt } \\
\text { alles. }\end{array}$ \\
\hline 4 & Bo. Ma. & I9 & $\mathbf{1}$ & $"$ & " & Spülung & " & Beschwerdefrei. \\
\hline 5 & $\mathrm{Ni}_{i}$ Ferd. & $5 \pi$ & 5 & $"$ & $"$ & $\operatorname{sen}$ & " & $\begin{array}{l}\text { Verträgt alles, ist } \\
\text { schmerzfrei. }\end{array}$ \\
\hline 6 & Oe. An. & $3 \mathbf{I}$ & 5 & $"$ & " & Spülung & $"$ & $\begin{array}{l}\text { Wohlbefinden, } \\
\text { keine Be- } \\
\text { schwerden. }\end{array}$ \\
\hline 7 & Nu. Phi. & 36 & $9 \frac{1 / 2}{1 / 2}$ & " & & Trocken & " & Beschwerdefrei. \\
\hline & |Scho. En. & 30 & $1 / 2$ & $"$ & B II & Spiilung & ., & $\begin{array}{l}\text { Beschwerdefrei, } \\
81 / 2 \mathrm{~kg} \text { Ge- } \\
\text { wichtszunahme. }\end{array}$ \\
\hline $\begin{array}{r}9 \\
10\end{array}$ & $\begin{array}{l}\text { Ha. En. } \\
\text { Stri. Jo. }\end{array}$ & $\mid \begin{array}{l}19 \\
23\end{array}$ & $\begin{array}{l}16 \\
14\end{array}$ & " & B"I & Spülung & 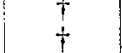 & $\overline{-}$ \\
\hline II & Ki. Ka. & 59 & 26 & $\because$ &, & Trocken & Heilung & Beschwerdefrei. \\
\hline 12 & Ca. Al. & 29 & 7 & , & & & $"$ & Beschwerdefrei. \\
\hline 13 & Za. Ma. & 30 & 4 & , & B II & Spïlung & 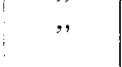 & $\begin{array}{l}\text { Verträgt alles, } \\
\text { schmerzfrei. }\end{array}$ \\
\hline 14 & Kri. Ro. & 24 & $2 \mathrm{I} 1 / 2$ & "' & $\begin{array}{l}\text { unilat. } \\
\text { Pylor.Aus- } \\
\text { schaltung }\end{array}$ & $\because$ & ", & $\begin{array}{l}\text { Vollständig } \\
\text { schmerzfrei, } \\
\text { verträgt alles. }\end{array}$ \\
\hline 15 & Kli. An. & 29 & 48 & & G E r. p. & $\because$ & $\dagger$ & - \\
\hline 16 & So. Tran. & 56 & I6 & ventric. & G E r.p. & " & $T$ & - \\
\hline 17 & De. Fr. & 25 & 23 & duod. & G E r. p. & 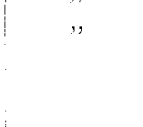 & Heilung & $\begin{array}{l}\text { Wohlbefinden, } \\
\text { keine Schmer- } \\
\text { zen, Gewichts- } \\
\text { zunahme } 6 \mathrm{~kg} \text {. }\end{array}$ \\
\hline 18 & Ka. Jo. & 27 & 6 & $\because$ & G E r.p. & 'Trocken & $"$ & $\begin{array}{l}\text { Ulcus pept. jejuni. } \\
\text { op. }\end{array}$ \\
\hline 19 & De. La. & $6 \mathrm{I}$ & 26. & ventric. & Naht & Spi &, & Beschwerdefrei- \\
\hline 20 & Os & 28 & 7 & duod. & G E r.p. & $T$ & $"$ & $\begin{array}{l}\text { Beschwerdefrei, } \\
\text { iBt alles. }\end{array}$ \\
\hline 21 & Ob. Ka. & 67 & 24 & & & Spi & $\dagger$ & - \\
\hline 22 & Fu. An. & 54 & 20 & ventric. & Naht & ren & $\dagger$ & $\ldots$ \\
\hline 23 & Tra. Jo. & 42 & 16 & duod. & $\mathrm{GE}$ & Sjülung & Heilung & Beschwerdefrei. \\
\hline 24 & Kn. Al. & 62 & 24 & $\mathrm{p}$ & Naht & $"$ & $\dagger$ & - \\
\hline 25 & Wi. An- & $27 \mid$ & 24 & ventric. & $\mathrm{G} \mathrm{E}-$ &. & Heilung & $\begin{array}{l}\text { Verträgt alle } \\
\text { Speisen, nie- } \\
\text { malsErbrechen, } \\
\text { Gewichtszu- } \\
\text { nahme } \mathrm{I2} \mathrm{kg} \text {. }\end{array}$ \\
\hline 26 & | Neu. Pet. & $60 \mid$ & 8 & duod. & " & ., & ", & $\begin{array}{l}\text { Ulcus reerd, ant. } \\
\text { Ulc. pept.? }\end{array}$ \\
\hline 27 & Stei. Wi. & 28 & 7 & ", & $"$ & " & " & $\begin{array}{l}\text { Wohlbefinden, } \\
\text { Gew.-Zunahme. }\end{array}$ \\
\hline
\end{tabular}


3 Magenulcera zur Anwendung. Um bei der Kleinheit des Ma. teriales einen besseren Uberblick über die Mortalität der Operationsverfahren gewinnen $z$ können, ist es nötig, dic indirekten, palliativen Methoden zusammenzufassen. Es ergeben sich dann für I3 Resektionen 2 Todesfälle (15,3 Proz.) für die I 4 indirekten Verfahren 5 Todesfälle (35.7 Proz.); zichen wir davon die 3 ein. fachen Übernähungen mit 2 Todesfällen ab), so verbleiben für die is Gastroenterostomien 3 üble Ausgänge. was einer Prozentzahl ron 27,2 entspricht. In Anbetracht der in der l.iteratur erwähnten höheren Sterblichkeit bei Duodenalulcera ist es vielleicht nicht uninteressant, unsere Erfolge bei diesen gesondert zu betrachten: von I I Resektionen starben 2 gleich 18 Proz., von den 9 Gastroenterostomierten ebenfalls 2 , das ist 22,2 I'roz.; also ziemlich gleiche Zahlen. Zusammengenonmen hatten wir bei 27 Fällen 7 Todes. fälle zu verzeichnen, was einer Sterblichkeit von nicht ganz 26 Proz. entspricht, einer Zahl, die nach dem bisher Bekannten sehr günstig wäre. Sie kommt am nächsten der von J. W. Struthers, der an der Hand von 90 Perforationen ( 72 Duodenal- i 8 Magenulcera) 20-25 Proz. errechnete. (Th. I) e m mer und Brunner geben 48 Proz. Mortalität an, Schülein 47,6 Proz., Brütt 42,5 P'roz.). Wir sind uns darüber klar, daß das hier zur Ver. fügung stehende Material doch vicl an klein ist. um verallgemei nernde Schlüsse zuzulassen.

Von den Resektionen sind g nach der Methode Billroth I, 3 nach Billroth II in der Modifikation Kronlein-Mikulicz und 1 quere Rescktion ausgeführt worden. Es mag an dieser Stelle betont werden, daß die mit den ersten Resektionen bei Ulcusperforationen erziclten günstigen Resultate $\mathrm{Habcrer}$ bestimmten, dieses Verfahren an einer größeren Reihe von Fällen auszuführen und ausführen zu lassen, um ihre augenblicklichen und Dauerresultate gegenüber denen anderer Methoden auszuwerten. Dabei spielte die Frage, ob die Resektion nach der oder jener. Methode zu beenden war, anfänglich eine untergeordnete Rolle, da die unserem Verfahren zugrunde liegende Überlegung (Haberer) darin ihren Kernpunkt hat, daß der Herd radikal entfernt werde. Die guten Erfolge die Haberer mit der Resektion der Licera nach der Methode Billroth I verzeichnete, erklären es auch, daß diese die besten Dauerresultate beim Ulcus ergebende Methode 
beim Ulcus perforatum zur Anwendung kam. Die diesbezügliche Mitteilung im Zentralblatt scheint $\mathrm{Br}$ üt ${ }^{1}$ ) entgangen $z \mathrm{u}$ sein.

Aus den oben angeführten Zahlen erhellt mit Deutlichkeit, $\mathrm{da} B$ wir bestrebt waren, die Magen-Darmvercinigung nach der Methode Billroth I durchzuführen; betont sei aber, daß wir das niemals erzwingen wollten, sondern überall dort, wo uns für cine exakte Serosanaht zu wenig Material zur Verfügung stand, nach Billroth II vereinigten. Erschien die Resektion mit Rücksicht auf die Ausbreitung der Peritonitis und den Allgemeinzustand als zu eingreifend, wurde einfach der l"bernähung die (iastroenterosto. mie angeschlossen. Nur dort, wo sich keine Notwendigkeit dafuir finden ließ, auch diese unterlassen. Der Vorwurf ciner Methode zuliebe Menschenleben aufs Spiel gesetzt zu haben, kann uns wohl nicht treffen.

Die beiden bei dem Resektionsverfahren zu beklagenden Todesfälle sind der Schwere ihrer Peritonitis anzurechnen, die vielleicht zur Zeit der Operation unterschätzt wurde.

Bei dem ersten, einem I9 jähr. Nanne, 16 Stunden nach Perforation, mit kleinem frequenten Puls und leichtem Meteorismus scheiterte der Versuch, die linsengroße an der Vorderwand des Duodenums befindliche Perforationsöfnung zu vernähen, an der Zerreißlichkeit der Iarmpartie. Da in dem infiltrierten brü. chigen Gewcbe keine Naht mohr hiclt, wurde die Resektion nach der Methode Billroth II - also mit Bürzelverschlul. am Duodenum in 3 Schichten - angeschlossen (Assistent Dr. Mitterstiller) Kochsalzspülung bei diffuser Peritonitis, vollständiger Peritonealschluß. Postoperativ standen die Zeichen der Toxinämic im Vordergrunde (hochgradige Lnruhe, hoher Puls); nach ganz kurzer Besserung kam er in der 3 . Nacht unter den Anzeichen einer neuerlichen Perforation zum Exitus. Die Obduktion crgab: diffus fibrinös-eitrige Peritonitis mit Bildung von abgesackten Eiterhöhlen subphrenisch. Thrombose der Arteria gastroduodenalis, eitrige Infilrattion des Bürzels mit Dehiszenz der Nähte, Thrombose der Arteria cocliaca am Abgang ron der . Iorta mit anämischen In-

1) In einem nachträglich in meine Ilände gelangten Separatum der Bruttschen Arbeit ist in Nachtrag bei der Korrektur II a bercrs Arbeit crwähnt. wodurch obige Behauptung richtig gestellt sei. Warum nicht in der Originalarbeit rermag ich nicht zu entscheiden. 
farkten in der Leber, akuter Milztumor. Magen-Darmanastomose suffizient. Die Beschaffenheit der Darmwand erwies sich in den tieferen Partien ebenso schwer geschädigt als in der Nähe des Ulcus; wenn auch bei der Vernähung des Bürzels keine Naht durchriß und das Gewebe sich scheinbar normal verhielt, so hatte die Klebekraft des Peritonum infolge der Entzündung schon schwer gelitten. Durch diese wie durch die Thrombose der Arteria gastroduodenalis, die wohl auch nur auf den schweren entzündlichen Prozeß zurückgeführt werden kann, war das Schicksal der Bürzelnaht entschieden. Deshalb erscheint es begründet den Fall dahin aufzufassen, daß die Schwere der Infektion den Ausgang bedingte. Der zweite betraf einen 23 jährigen Mann, der 14 Stunden nach dem Schmerzanfall mit starkem Meteorismus und ausgesprochener Flankendämpfung rechts, bei recht verfallenem Gesamteindruck zur Operation kam.(Doz. Dr. Chiari). Die Umgebung der erbsengroßen Perforationsöffnung ist derartig schwielig verändert, daß ein Nahtverschluß nicht exakt durchführbar ist. Daher Resektion des Ulcus und präpylorischen Magenteiles und Magen-Darmvereinigung nach der. Methode Billroth I. Dabei besteht schwerste Peritonitis, die subphrenisch ca. I Liter Eiter entleeren läßt und nur längs des Colon ascendens bis in die Cöcalgegend herunterzureichen scheint. Drainage des Subphreniums von vorne und seitlich am scheinbar unteren Ende des Abszesses. Nach trockener Reinigung der Bauchhöhle (da die linke Seite frei von Eiter war) primärer Wundschluß. Leichte Besserung, am 3. Tage Stuhl, keine Empfindlichkeit des Abdomens. Am 4. Tage plötzliche Verschlimmerung und Exitus. In autopsia werden allgemeine Sepsis, lobulär pneumonische Herde in beiden Lungen, eitrig-fibrinöse Pleuritis beiderseits, akuter Milztumor und eine auf die rechte Seite und den Douglas beschränkte Peritonitis purulenta festgestellt. Anastomosennähte vollkommen dicht. Drainage des Subphreniums von Erfolg, da sich nur mehr spärliche Reste von fibrinösen Belägen auf der Oberfläche der Leber fanden.

Wenn es auch hier gelungen war, die Bauchhöhle möglichst von Eiter zu befreien, so war der schlechte Ausgang doch nicht aufzuhalten; wohl deshalb, weil die Invasion des Körpers mit Eitererregern zu weit fortgeschritten und dieselben schon vor 
dem Einsetzen der Therapie in Blut- und Lymphbahn übergetreten waren. Die vorübergehende Besserung und Weichheit des Leibes, das Wiedereinsetzen der Peristaltik können sicher nicht als dagegen sprechend angeführt werden, denn so manche Peritonitis, die sich im postoperativen Verlauf nach einer aseptischen Laparotomic (Nahtinsuffizienz) entwickelt, kann unter so geringen, klinischen Erscheinungen einhergehen, da $B$ man erst daran denkt, wenn der Puls auffallend mit der 'Temperatur disharmoniert. In beiden Fällen ist der üble Ausgang wohl der Ausdehnung und Schwere der Infektion zuzuschreiben und man darf - so scheint es - mit Recht annehmen, daß jede andere operative Maßnahme, wäre sie auch noch so kurzdauernd gewesen, daran nichts hätte ändern können. Auch darauf haben beide Fälle aufmerksam gemacht, daß Metcorismus, ein übles Anzeichen vor jedem intraperitonealen Eingriff, bei der Perforationsperitonitis ganz besonders zu berücksichtigen ist (Sch nitzler). Schließlich gehörten beide schon den Spätfällen an, wenn die Grenze, innerhalb welcher von Frühfällen gesprochen werden darf, mit 12 Stunden festgelegt wird.

Die bei den 14 indirekten Methoden verzeichneten 5 'Todesfälle (nach I6, 24 [2 mal], 26, 48 Stunden) verteilen sich so, daB 2 davon auf dic 3 einfachen Übernähungen des Ulcus, die übrigen 3 auf Übernähung plus Gastroenterostomie entfallen. Es starben 4 an der als Folge der Perforation entstandenen nicht mehr beeinflußbaren Peritonitis, I an Peritonitis infolge Nichthaltens der Magennaht (mit Gastroenterostomie). Von den 2 ohne entlastende Gastroenterostomic vorgenommenen Übernähungen kam der eine bereits am selben Tage infolge der Schwere der Infektion zum Exitus, der andere erst nach 13 Tagen an der diffus eitrigen Peritonitis, die zu zahlreichen abgesackten Abszeßbildungen zwischen den geblähten Darmschlingen besonders unter dem Zwerchfell geführt hatte. In beiden Fällen waren die Nähte suffizient. Die Gastroenterostomic wurde vermieden, um den Eingriff bei dem schwer darniederliegenden Allgemeinzustand und der langen Dauer des Bestehens der Peritonitis (20, 24 Stunden) nicht über Gebühr zu verlàngern und andererseits, weil durch die Übernähung keine nennenswerte Stenose erzeugt war. Beachtenswert bleibt noch, daß der eine Fall gespült, der andere trocken gereinigt wurde; der "Gespülte" zeigte anfänglich eine Besserung seiner perito- 
nitischen Symptome, wurde aber nit dem zurückgebliebenen Reste nicht fertig, hatte seine Widerstandskraft crschöpft und erlag seiner Peritonitis. Der "Gewischte" starb schon nach 24 Stunden an der Schwere seiner Infektion. Diese beiden Fälle bestätigen die Worte Noctzls, ,daß wir bei einmal eingetretener bak. terieller Infektion nach der Perforation den lebensrettenden Erfolg nicht mehr in der Hand haben".

Die mit Gastroenterostomic kombinierte Übernähung der Ma. gengeschwürsperforation, die durch Insuffizienz der Naht nach I6 Tagen zum Exitus kam, lag folgendermaßen: Op. (Assistent I)r. Paul): Das sattelfürmig der kleinen Kurvatur aufsitzende Ulcus war ins Pankreas penetriert, die Vorderfläche des Magens weit infiltrierend, so daß3 das Heranziehen normaler Magenwand nur unvollkommenen Verschluß der Öfnung brachte. Es wurde deshalb die Ilombierung mit dem rechten Leberlappen (einem Schnürlappen) zur Unterstützung herangezogen. Durch Io Tage währte die Dichtigkeit des Verschlusses, bis scheinbar Nahtabszesse den schützenden Deckel lösten. Eine Exzision kam bei der Größe der Infiltration gar nicht in Frage, ja selbst dic erst kürzlich von $B$ ü $r k$ angegebene Technik zur Gestaltung ciner

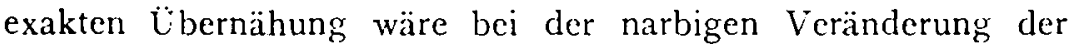
Vorder- und Hinterwand undurchführbar geblieben, da cine ausreichende Verschiebung der Magenwand infolge der breiten Penetration ins Pankreas unmöglich war. Der 3. einfache Übernähungsfall (ohne Gastroenterostomic) 26 Stunden nach der Per. foration heilte glatt ohne Stenose des doch ziemlich cingeengten Duodenums. Es erscheint uns deshalb nicht statthaft, das Nicht. halten der Naht so apodiktisch auf das Unterlassen der Castroenterostomic zurückführen zu wollen, wie das in der Literatur des öfteren zu lesen ist. Immer bleibt die Peritonitisbehandlung das Wichtigste und diese ist am erfolgreichsten, je früher wir die Fälle in Behandlung bekommen. Diese Erfahrung veranlaßte die Trennung der Fälle in Spät- und Frühfälle. Während die einen (Demmer, Schülein usw.) mit 9 Stunden die Frühfälle begrenzen, erweitern andere (Pctrén, $\mathrm{Na}$ umann, Brüt t) diesen Zeitraum dadurch, daß sie erst nach I 2 Stunden von Spätfällen sprechen. Selbstverständlich wird eine Zusammenstellung unter diesem Gesichtspunkte genauere Aufschlüsse über die Erfolge und 
den Wert der einzelnen Verfahren in der Peritonitisbehandlung bringen. Umstehende Tabelle mag unsere Erfolge beleuchten. Innerbalb der ersten | to Resektionsfällen o Todesfälle $=$ o Proz. Mort. 12 Stunden bei

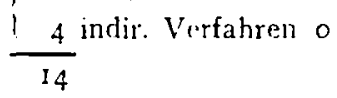

Naci 12-26 Stunden | 3 Resektionen bei $\frac{1 \text { 10 Indirekten }}{13}$

$$
\begin{array}{rlr}
2 & . & -66,6 \\
5 & \therefore & =50 \\
& & =53,8
\end{array}
$$

Ein genaues Eingehen auf die anläßlich der Nachuntersuchung erhobenen Befunde erscheint uns nicht nur gestattet, sondern sogar geboten, da sich vielleicht doch daraus Anhaltspunkte für die Beantwortung der Frage ergeben, ob die Resektion beim perforierten Ulcus in Betracht gezogen werden darf, wann sie anzuwenden ist und welches Verhältnis bezüglich augenblicklicher Mortalität und Dauerresultaten gegenüber den indirekten Methoden bestehe. Dic Resektionen müssen bei Ulcusperforationen in Betracht gezogen werden, sobald ihre Dauerresultate bessere sind als die der bisherigen operativen Maßnahmen -, so daß dadurch, wie Brït sich in seiner Arbeit im Zentralblatt 1921 ( $\mathrm{Nr} .38$ ) ausdrückt, „eine eventuelle höhere Operationsmortalität wieder ausgeglichen wird". In dieser Arbeit berichtete er über 12 nach ähnlichen Indikationen operierte Ulcusperforationen, von denen er nur einen an seiner durch hämolytische Streptokokken bedingten Peritonitis verloren hat. Auch Brütt legte den größten Wert auf den primären Schluß der Bauchwunde, nachdem der Herd „restios" entfernt wurde. Fir resezierte nach Reichel-Hofmeister und entfernte den Eiter je nach der Ausdehnung des Prozesses durch Spülen oder Wischen. Wenn er dabei sagt, daß es sich um, ,beg innende diffuse Peritonitis handelte, so müßten wir hervorheben, daß in der Hälfte unserer Fälle - natürlich die bald nach der Perforation eingelieferten ausgenommen - alle Anzeichen für eine a usgebildete, gan $z$ dif $\mathrm{g}$ se Bauchfellentzündung angetroffen wurden, was für die Beurteilung des Erfolges der eingeschlagenen Therapie von Bedeutung sein muB. Die Gefäßinjektionen am Darme waren in diesen Fällen intensivst ausgebildet, die Veränderungen des Peritoneums und der Mesenterien unzweideutig vorhanden und Eiter nicht nur an den beiden Subphrenien und im Douglas, sondern überall zwischen den Darm- 
schlingen zu finden. Das nach Einleiten der Kochsalzspülung ablaufende Spülwasser war immer eitric und klärte sich erst langsam.

Sind günstige Erfolge auch noch 26 Stunden nach der Perforation zu: erzielen, so kamen, wie aus der Tabelle zu ersehen ist, Ulcusperforationen, die viel kürzere Zeit nach dem Durchbruch operiert wurden, trotz gleicher Behandlung, ohne daß Nahtinsuffizienz oder ein sonstiger technischer Fehler als Ursache anzuschuldigen gewesen wäre, unaufhaltsam zum Exitus. Hier war die Allgemeinschädigung durch den Infektionskeim so rasch und so groß, daß keine Widerstandskraft übrig blieb. die den Kampf mit den zurückgebliebenen Keimen nach Reinigung erfolgreich hätte aufnehmen können. Die Art und Virulenz der Keime wird aus. schlaggebend bleiben. Brït hat durch systenatische bakterio. logische Intersuchungen, - clie leider bei uns unterlassen wurden

außer der Beobachtung, daß selbst noch nach 18 Stunden die Flüssigkeit an- und aërob steril bleiben kann isiehe auch Prader), in der Mehrzahl der Falle aus dem Exsudat einen grün wachsenden Streptocorcus in Reinkultur züchten können, dessen Verhältnis zum Llcus, zur l'erforation und zum (irade der Peritonitis noch nicht geklärt erscheint. Leider ist eine sichere Nethode, die uns rasclı über die l'athogenitït der vorhandenen Bakterien vor dem operativen Eingriff unterrichten kann, noch nicht bekannt, und klinisch kein I nterschied zu erfassen.

Im großen und zäanzen künnen wir unser Verfahren der P'eritonitisbehandlung nach den verhältnismäßBig günstigen Ergebnissen. dif- durch B rï tts Beobachtungen bestätigt werden. wärm. stens empfehlen, und da die Dauerresultate ebenfalls recht erfreulich sind, zur Nachprüung auffordern.

Es mag auch kurz erwähnt werden, daß alle unsere Magenuperierten mit der Weisung entlassen wurden, die bekannte Säure-, lett- und Germspeisen meidende Jiät durch ein Jahr strenge einzuhalten und nach Fleischgenuls Natr. bicarb. zu neh. men. Zumeist crählten die wegen Perforation Resezierten, daß sie Speisesoda bald wegließen, nach längerer oder kürzerer Zeit keine Auswahl in der Nahrung trafen und zu schwerer Kost bald übergingen, - ja selbst Wein wurde ohne Schaden vertragen. Nur cin ganz kleiner Teil hat auch nur aus (iewissenhaftigkeit 
die Vorschriften strenge befolgt. Über Aufstoßen, Brechreiz, Sodbrennen usw. konnte von keinem Klage geführt werden. Übereinstimmend wird von ihnen die regelmäßige ohne jedes Abführmittel erfolgende Stuhlentleerung gemeldet; ganz besonders wird dies von denjenigen betont, die vorher an Obstipation litten. Von seiten des Abdomens bestanden keine unangenehmen Sensationen, wie intermittierend auftretende Blähungszustände, Schmerzen oder Druckgefühl. Die meisten von ihnen sind wieder in ihrem schweren, anstrengenden Berufe tätig und haben trotzdem oft ganz bedeutende Gewichtszunahme zu verzeichnen, die bei denen größer ist, die vorher in der Ernährung vorsichtig sein mußten. Subjektiv also ganz ausgezeichnete Befunde!

Die Magensaftuntersuchungen nach Probefrühstück ergaben in den meisten Fïllen das Fehlen freier Salzsäure oder nur geringe Werte derselben. Der Vergleich mit den Operationsgeschichten hat nun ergeben, daß diese Werte nicht immer parallel gehen mit den Ausdehnungen der Resektion kardialwärts, so daß bei Entfernung großer Magenpartien manchmal relativ höhere $\mathrm{HClmengen}$ vorhanden waren, als bei kleineren Resektionen. Was die Ausdehnung der Resektion betrifft, so wurde in der Mehrzahl der Fälle ,hoch über die Magenmitte" hinaufgegangen, , $2 / 3$ des Magens" entfernt, weil derselbe „groß, hypertroph und ptotisch“ erschien. Dabei wurden an der großen Kurvatur alle Gefäße mit Ausnahme der Gastricae breves unterbunden. In dem kleineren Teil der Fälle wurde bald dies- bald jenseits der Magenmitte abgesetzt und nur in 2 Fällen von Duodenalulcera der antrale Teil des Magens reseziert. Dadurch, daß ich fast sämtlichen Resektionen beiwohnen konnte und immer Gelegenheit hatte den resezierten Magenteil im frischen Zustande zu sehen, war mir die Beurteilung des Verhältnisses zwischen $\mathrm{HCl}$ Wert und Resektionşröße erleichtert. Dieses scheinbare Mißverhältnis kann aber vielleicht durch den Hinweis auf die Verschiedenheit der Aziditätswerte unter Gesunden und Ulcusträgern, sowie durch die bei den einzelnen Individuen verschieden hochgezogene Grenze, bis zu welcher $\mathrm{HCl}$ Drüsen vorkommen, erklärt weriden.

Dabei konnte in keinem Magensafte der nach Billroth I Operierten, Galle oder Pankreassaft gefunden werden, was wohl be- 
weist, daß diese den natürlichen Verhältnissen am nächsten kommende Magen-Darmvereinigung mit der Zeit einen guten Abschluß gegen das Duodenum erhält. In der ersten Zeit post operationem wurde auch von sämtlichen nach Billroth I Resezierten, nach Gemuß von Hefe- und fettzubereitenden Speisen über Unbehagen im Magen gleich nach der Mahlzeit und häufigem Stuhlgang Klage geführt. Diese Zustände verloren sich im Verlaufe des 2. und 3. Monates gänzlich. Diese Erscheinung wäre unserer Meinung nach vielleicht mit der anfänglich bestehenden Sturzentleerung des Magens in Zusammenhang zu bringen, durch die der Darm überlastet zu werden scheint. Die röntgenologisch nachgewiesene Sturzentleerung ${ }^{1}$ ) ist bei der Nachuntersuchung nicht mehr vorhanden, sondern hat einer langsameren rhythmischen Platz gemacht. Röntgenologisch ${ }^{2}$ ) fehlte jede Hypersekretion (bei nach Billroth I Operierten); Wandveränderungen und Druckschmerz waren nie zu konstatieren.

Die Gastroenterostomierten fühlten sich subjektiv im großen und ganzen keineswegs schlechter als die Resezierten. War auch bei diesen der Übergang zur gemischten Kost ohne weitere Störungen erfolgt, so konnten mit Ausnahme an 2 Fällen, subjektive Beschwerden oder klinische Veränderungen nicht nachgewiesen werden. Röntgenologisch ergab sich die Gastroenterostomie gut funktionierend: zuführende Schlinge vom Pylorus her sehr schlecht, von der Einpflanzungsstelle vom Magen her gar nicht fühlbar. Der Mageninhalt nach Probefrühstück ergab größtenteils Zahlen normaler Werte; Galle war in allen Fällen vorhanden.

Komplikationen verratende Befunde konnten nur an 2 Gastroenterostomierten erhoben werden.

Der eine ist ein 6o jähriger Mann dem vor ca. I Jahre wegen eines perforierten Duodenalgeschwüres außer der Übernähung eine G. E. angelegt wurde; Op. (Ass. Dr. O. $\mathrm{M}$ a i er), den Eingriff komplizierte damals eine Hernia encystica, deren Bruchsack eitergefüllt war. Kochsalzspülung, primärer WundschluB, nach 5 Wochen Heilung. Anläßlich seiner Vorstellung behufs Nachuntersuchung klagte er über „Magenschmerzen“ und Erbrechen. - Es bestand ein Druck-

I) v. H a b e rer. Zentralbl. f. Chir. I92 I, S. 847 .

2) Die Röntgenuntersuchungen wurden im Zentralröntgen-Institut Dozent Dr. Sta unig ausgeführt, dem auch unser besonderer Dank gebührt.

Deutsche Zeitschrift f. Chirurgie, r72. Bd. 
punkt in Epigastrium wie auch rechts vom Nabel in dessen Höhe. Die Schilderung der Beschwerden Iieß an cine anatomische Veränderung denken, weshalb der Mann für den nüchsten Tag nüchtern hereinbestellt wurde. Allein er ist bis jetzt trotz wicderholter freundlichster Aufforderung nicht mehr erschienen. Es ist nicht ausgeschlossen, daß es sich bei ihm um ein Rezidiv im Duodenum oder um ein Ulcus pepticum handelt, jedoch leider nicht möglich, Ge. naueres anzugeben.

Der II. Fall 6 Stunden nach der Perforation eines kallüsen Duodenalulcus, mit G. E.r. p. und Übernährung behandelt. Operateur: Ass. Dr. Paul (Dez. I919). Exsudat wurde trocken ausge. wischt; konnte natch 17 Tagen bereits beschwerdefrei entlassen werden. Während der nächsten Zeit ging es ihm tadellos. Er hatte guten Appetit, vertrug alle Speisen und nahm an Gewicht zu. Dieser günstige Zustand hiclt bis Anfang dieses Jahres an, zu welcher Zeit sirh nach dem Essen Schmerzen einstellten, die ihn zwangen sich eine gewisse Beschränkung in der Nahrung aufzulegen. - Die Schmerzen in der Mittellinic und über dem Nabel beginnend waren zicmlich heftig und strahlten gegen die Flanken, mehr gegen die linke aus. Ihr Auftreten war ein ziemlich regelmäßiges, und zwar mittags eine Stunde nach dem Essen und abends zwischen 6 und $\rightarrow$ Uhr. Dabei bestanden kein Erbrechen, kein Aufstoßen, unregel. mäßiger Stuhlgang, jedoch ohne Blutbeimengung . Klinisch ließ sich ein Schmerzpunkt 3 Querfinger links und rechts vom Nabei feststellen. -.. Dic Säurewerte ergaben $35 \mathrm{Ges}$.Azid., $56 \mathrm{HCl}$, Benzidin im Magensaft positiv. Röntgenologisch war Hypersekretion und rasche Entlcerung des Kontrastmittcls zu sehen. - Die Einpflanzungsstelle der G. E. und ein Punkt $2 \mathrm{~cm}$ darunter blieb druckschmerzhaft. Nach 3 Stunden kein Rest - Vcrdacht auf Ulcus pepticum. Dic Laparo tomic (Prof. v. H a be rer) stellte unterhalb der antiperistaltisch an. gelegten G.E. ein müchtiges Ulcus pepticum an der abführenden Schlinge (Mesenterialansatz), verlötet und durchgebrochen in die Wand des Colon transversum fest. - Das übernähte Duodenalulcus war ausgeheilt und nur mehr eine kleine $1 / 2 \mathrm{~cm}$ lange feine Narbe sichtbar. Freilegen der G.E. Durchtrennung der Jejunalschlinge ober- und unterhalb derselben End-zu-Endnaht des Jejunum. Hierauf Ausschälen des Ulcus pept. aus dem Colon transversum, was ohne Eröffnung des Darmlumens möglich war. Ubernähung des Colon. Resektion des Magens vom Duodenum unterhalb des Geschwüres angefangen bis über die G.E. hinaus, Magendarmvereinigung nach Br. Patient, der am 16. XI. 192I opericrt wurde, ist am 7. XII. 1921 geheilt entlassen worden. -

Zu diesem Falle düfte sich jedes weitere Kommentar erübrigen. -

Das Ergebnis dieser Nachuntersuchungen ist schon aus dem Grunde recht verläßlich, als die größere Zahl der Einberufenen 
sich persönlich vorstellte, die übrigen durch ihren Hausarat den an sie gerichteten Fragebogen erledigen ließen. Dadurch erhielten wir Kenntnis von dem Befinden von ro Resektionsfällen (einer war an einer interkurrenten Krankheit gestorben) und 9 Gastro. enterostomierten. Wenn auch die meisten Fälle über ein und zwei Jahre post operationem zurückliegen, so sind wir uns klar darüber, daß unsere jetzigen Ergebnisse in dem einen und anderen Punkte zu einem noch späteren Zeitpunkte nachgeprüft, Verschiebungen erleiden können!

Bevor wir die gewonnenen Beobachtungen zusammenfassen, möchten wir noch 4 Fälle von sogenannter subakuter Perforation nicht unerwähnt lassen, die alle wegen fast plötzlicher Verschlimmerung ihres gewöhnlich schon jahrelang bestehenden Leidens ins Spital kamen und operative Behandlung forderten. Sie hatten alle das gemeinsam, daß nach Eröffnung der Bauchhöhle entweder schon bei belassenem Situs der Eingeweide oder nach vorsichtiger Darstellung des Ulcus, jedenfalls olne den Magen irgendwie nennenswert vorzuziehen, das nur durch einen lïbrinpfropf verschlos. sene Ulcus einril, oder dic durch Fibrin zustandegekommene lose Verlötung, mit dem Nachbarorgan getrennt wurde; bemerkenswert waren dic reichen, fibrinösen Auflagerungen in dem das Ulcus tragenden Magendarmteil, sowic die hochgradig ausgebildeten ent. zündlichen Erscheinungen in der weiteren Umgebung. Lange Ulcusanamnese, Periodizität der Schmerzen, plötzliche Verschlim. merung nach vorheriger stärkerer Abmagerung, einmal mit erstmaliger stärkerer Blutung, sind die den meisten gemeinsamen Merkmale. In einem Falle jedoch war außer einem seit $5 \mathrm{Mo}$ naten bestehenden Hungergefühl anamnestisch nichts feststellbar! 3 mal lag ein Ulcus duodeni vor mit ungewöhnlich tiefem Sitz. $(6$ und $4 \mathrm{~cm})$. In einem Falle waren 2 Ulcera vorhanden; das perforicrende saß $\mathrm{im}$ Duodenum das kleinere ebenfalls kallös nahe dem Pylorus. Das 4. betraf ein Ulcus der kleinen Kurvatur des Magens. Dic in allen 4 Fällen ausgeführte Resektion (sämtlich von Prof. v. Haberer operiert) brachte Heilung. Die Ergebnisse der Nachuntersurhung unterschieden sich in gar nichts von den oben angeführten.

Aus den Ausführungen ergeben sich folgende Schlüsse:

Die Resektionsmethode darf beim Ulcus ren. 
tric. et duodeni perforatum in Anwendung kom. men, wobei mehr die Berücksichtigung des All. gemeinzustandesals dienach der Perforationver. strichene Zeit entscheidet.

Die bei der Resektion beobachteten Opera. tionsresultate sind günstig.

Die an dem Material der Innsbrucker Klinik angestellten Nachuntersuchungen ergeben bes. sere Dauerresultate beider Resektion, als beiden indirekten Methoden, nach denen z. B. Ulcera pep. tica auftreten können.

\section{Literaturverzeichnis.}

I. Baurmann. Deutsche Zeitschr. f. Chir., Bd. 154. S. 4i.

2. Benett. The Lancet 1896, Vol. II, p. 310 .

3. Bra un. Zentralbl. f. Chir. 1897, 27.

4. Brunner, C. Bruns' Beitr., Nr.40. Arcl. f. klin. Chir., Bd. 31, S. 740

5. Brunner, Fr. Deutsche Zeitschr. f. Chir., Bd.69, S. Ior.

6. Brütt. Bruns Beitr., 123 .

7. -. Zentralbl. f. Chir. 1921, Nr. 38.

8. B u rk. Zentralbl. f. Chir. 1920, 25.

9. Chiari, O. M.. Bruns' Beitr., Bd. 120, H. 2.

10. Demmer, Th. Bruns' Beitr., Bd.n1, S. 4 oo.

II. Dewes. Deutsche med. Wochenschr. 192I, Nr. 20.

12. E berle. Zentralbl. f. Chir. 1920, Nr. 45.

13. Eunike. Deutsche med. Wochenschr. 1919, Nr. 28.

14. 'H a berer, v. Wiener klin. Wochenschr. 1919, Nr.16, 1920, Nr. 27.

15. H rom a da. Wiener klin. Wochenschr. 1921, 36 .

16. Kroher. Deutsche Zcitschr. f. Chir., Bd. 134.

17. Kroiss. B3runs' Beit.r., 67.

18. Markoni. Wiener med. Wochenschr. 1920, 25.

19. Massari. Wiener klin. Wochenschr. 1920, 12.

20. Möller. Ref. Zentralbl. f. Chir. 1917, 1.

21. Noetzel. Bruns' Beitr., 47, 337.

22. Petrén, G. Bruns' Beitr., 72, S. 319.

23. Prader. Wiener klin. Wochenschr. 1920, 50.

24. R o ed elius. Bruns' Beitr., 92, S. 277.

25. Rosentha1. Bruns' Beitr., IIo, S. 551.

26. Schoemaker. Zentralbl. f. Grenzgeb., Bd. 18, H. I.

27. Schülein. Deutsche Zeitschr. f. Chir., 16ı, S. 242.

28. S chwarzmann. Wiener klin. Wochenschr. 1920, 22.

29. Struthers, J. W. Ref. Zentralbl. f. Chir. 1921, 2. 